\title{
BMJ Open Impact of mobile applications on adherence to cancer treatment: a systematic review and meta- analysis protocol
}

\author{
Kleyton Santos Medeiros, ${ }^{1}$ Janice França Queiroz, ${ }^{1}$ Michelly Nóbrega Monteiro, ${ }^{1}$ \\ Weruska Alcoforado Costa, ${ }^{1}$ Ricardo Ney Cobucci, ${ }^{2}$ Beatriz Stransky, ${ }^{3}$ \\ Ana Katherine Gonçalves ${ }^{1}$
}

To cite: Medeiros KS,

Queiroz JF, Monteiro MN, et al. Impact of mobile applications on adherence to cancer treatment: a systematic review and metaanalysis protocol. BMJ Open 2019;9:e027246. doi:10.1136/ bmjopen-2018-027246

- Prepublication history for this paper is available online. To view these files please visit the journal online (http://dx.doi org/10.1136/bmjopen-2018027246).

Received 21 December 2018 Revised 14 September 2019 Accepted 25 September 2019

Check for updates

(c) Author(s) (or their employer(s)) 2019. Re-use permitted under CC BY-NC. No commercial re-use. See rights and permissions. Published by BMJ.

${ }^{1}$ Health Science Postgraduate Program, Universidade Federal do Rio Grande do Norte, Natal, Brazil

${ }^{2}$ Universidade Potiguar Unidade Salgado Filho, Natal, Brazil

${ }^{3}$ Universidade Federal do Rio Grande do Norte, Centro de Tecnologia, Natal, Brazil

Correspondence to Dr Ana Katherine Gonçalves; anakatherine_ufrnet@yahoo. com.br

\section{ABSTRACT}

Introduction The number of patients taking oral chemotherapy is increasing around the world. It is essential to maximise the adherence to oral chemotherapy to improve the overall survival and life expectancy of the patients. In this systematic review and meta-analysis, we aim to evaluate the effectiveness of mobile applications in improving the adherence to oral chemotherapy and adjuvant hormonal therapy in cancer survivors.

Methods and analysis MEDLINE, Embase, LILACS, clinicaltrials.gov, Scopus and the Cochrane Central Register of Controlled Trials will be searched for randomised or quasi-experimental studies published between January 2009 and July 2019. This systematic review and meta-analysis will include studies investigating the use of mobile applications by cancer survivors to aid adherence to oral chemotherapy and adjuvant hormonal therapy. Patient education, reminder tools, calendars, pillboxes and electronic reminders will not be evaluated. The primary outcome will be the improvement in adherence to anticancer drugs. The secondary outcomes will be an improvement in the overall survival and life expectancy, improved quality of life and control of cancerrelated symptoms. Three independent reviewers will select the studies and extract data from the original publications. The risk-of-bias will be assessed using the Cochrane risk-of-bias tool. Data synthesis will be performed using the Review Manager software (RevMan V.5.2.3). To assess heterogeneity, we will compute the $I^{2}$ statistics. Additionally, a quantitative synthesis will be performed if the included studies are sufficiently homogenous.

Ethics and dissemination This study will be a review of the published data, and thus, ethical approval is not required. Findings of this systematic review will be published in a peer-reviewed journal.

PROSPERO registration number CRD42018102172.

\section{INTRODUCTION}

\section{Description of the condition}

About 25\% of the new antineoplastic agents under development are estimated to be oral drugs. Notably, the number of oral chemotherapeutic drugs will be more than doubled over the next few years. ${ }^{1-3}$ Compared with
Strengths and limitations of this study

- This systematic review and meta-analysis aims to combine the results of different studies that have comparable effect sizes and can be computed.

- Three reviewers will independently select the eligible studies, extract data without different variables and assess the risk-of-bias.

- There is a possibility that we get a small sample size and a limited number of studies; this may influence the validity and reliability of the findings.

- Different types of mobile applications may cause considerable heterogeneity that could limit generating convincing conclusions.

- Despite these limitations, the findings of this systematic review and meta-analysis may suggest whether mobile applications or other approaches are more useful in improving the adherence to oral chemotherapeutic treatment.

intravenous therapy, oral therapy is more convenient, faster and easier to administer, and requires fewer clinic visits and hence, preferred by the patients. ${ }^{45}$ Additionally, oral therapy can provide a feeling of control over treatment, reduce the interference of treatment with work and social activities, and eliminate the requirement of travelling to an infusion clinic and the discomfort of inserting an intravenous line. ${ }^{2}$ Once an antineoplastic agent is ordered, the administration is the responsibility of the patient. ${ }^{5}$ However, patients and clinicians are facing new challenges in managing adherence to these oral therapies. ${ }^{6}$

Most patients attempt to adhere to the treatment according to the prescription, nevertheless, adherence continues to be a problem. It is difficult to obtain a reliable estimate of adherence to oral antineoplastic therapies from the literature. This is because the few intervention studies that have been 
conducted on treatment adherence have notable methodological concerns. Thus, there is limited evidence to promote treatment adherence in patients with cancer. ${ }^{6}$ Moreover, studies on non-adherence to treatment and pharmacological limitations are inadequate. ${ }^{3}$

Hershman et $a l^{7}$ showed that the interventions to enhance the psychosocial well-being of patients should be evaluated to increase treatment adherence. Furthermore, the authors explained that adherence to therapy has been reported to be associated with belief in the efficacy of the drug and with belief in the benefits of taking prescribed drugs; and a high level of cancer-specific emotional distress was associated with subsequent nonadherence to treatment. Another study suggested that poor physician-patient communication, negative feeling regarding the efficacy of the drugs and fear of toxicities were associated with failure to initiate the therapy. ${ }^{6}$

In a systematic review, Greer et at assessed the interventions to improve adherence to oral antineoplastic therapies in patients with various malignancies. These interventions included educational support, monitoring treatment, pharmacy-based programme, counselling programme, and use of pre-filled pillboxes and automated voice response systems. Nevertheless, most of the studies included in this systematic review had a high riskof-bias due to non-randomised designs, small sample sizes, subjective assessments of adherence and missing data. In another systematic review of interventions to promote adherence to oral antineoplastic therapies, the investigators drew similar conclusions, as problems nonadherence to treatment. ${ }^{8}$

A variety of education, symptom management and reminder-based interventions, which involve faceto-face interactions, phone calls and texting short message service (SMS) have been developed and tested. However, the effectiveness of these interventions remains inconclusive. $^{9-11}$

\section{Description of the intervention}

The American Society of Clinical Oncology/Oncology Nursing Society recommends educating patients on the administration of oral chemotherapy. ${ }^{12}$ This includes the following:

- The storage, handling, preparation, administration and disposal of oral chemotherapeutic drugs.

- Concurrent anticancer treatment and supportive drugs/measures (when applicable).

- Possible drug-drug and drug-food interactions.

- A plan for missed doses. ${ }^{12}$

The oncology nurses can use tools and technology to assist with education, which may promote treatment adherence. In this context, patient education programme and physical devices, such as pillboxes and glowing pill bottles have been developed. Additionally, computer and mobile applications have paved the way for electronic reminders, such as calendars, text messaging, and alarms. 51314
Mobile applications are softwares that support a wide range of function of the mobile phone, including television, telephone, video, music, word processing and internet service. ${ }^{15}$ The first drug reminder application was developed in $2009 .{ }^{56}$ Mobile applications have several advantages compared with other interventions; this include simple and easy use, often in an automated fashion using a computerised programme. ${ }^{6}$ Thus, mobile applications may be used to encourage healthy lifestyles while monitoring, tracking, collecting, and transmitting data in real-time, facilitating the doctor-patient communication and increasing the cooperation between the patient and health professionals. ${ }^{7}$

Several techniques may increase treatment adherence, the most effective being behavioural approaches. However, there is no consensus on which behavioural techniques (such as specific goal-setting, self-monitoring and social comparison) are most effective in promoting treatment adherence. ${ }^{7}$

With the ever-increasing use of smartphones and development of potentially effective behavioural intervention technologies, scientists may be able to collect data in realtime in a real-world setting. Additionally, researchers are able to optimise the delivery of behavioural interventions and collect data with minimal burden to the patient and provider. ${ }^{11}$ Recently, a review suggested that adopting mobile technologies to deliver accessible interventions could improve health behaviours in patients with cancer. ${ }^{13}$

\section{Intervention mechanisms}

Adherence remains a complicated issue in the treatment of chronic diseases. ${ }^{8-14} 16-18$ In this context, the benefits of using technology, even in the form of a simple text message, have been recognised. ${ }^{19}$ This may improve adherence to the prescribed dosage, with an increase in adherence rates ranging from $50 \%$ to $67.8 \% .^{14}$ Mobile applications are suitable for delivering various educational and behavioural interventions while enabling caregivers and health professionals to monitor the patients' drug consumption patterns. ${ }^{10}$

\section{Why it is important to perform this review}

The traditional interventions to improve long-term treatment adherence are complex and not widely used. There is a widespread need for innovations that would provide convenient and feasible techniques to help patients remain adherent to the treatment. ${ }^{18}$

Currently, the average rate of non-adherence to oral anticancer therapy is estimated to be around $21 \% .{ }^{4}$ This demonstrates that poor adherence is a barrier to completing the treatment. ${ }^{1819}$ Non-adherence is complex and systemic; moreover, when at home, there is no professional method to know whether patients are correctly taking the drugs as prescribed. Oral regimens may be associated with complicated dosing schedules; additionally, due to food-drug interactions treatment adherence may become difficult. In busy clinics, patients may be given documents about the new $\operatorname{drug}(\mathrm{s})$; however, the 
time available for one-on-one interaction may not be sufficient. ${ }^{5}$ Ensuring patient adherence to a treatment that involves self-administration is a challenge faced by healthcare providers. ${ }^{20}$ Many factors can affect treatment adherence: lack of understanding regarding proper administration, complex dosing regimens, administration of other potentially interacting drugs, the timing of drug doses with respect to food intake, cost of the drug and unpleasant side effects. Furthermore, common health conditions of the patients, such as visual and cognitive impairment, memory deficits or forgetfulness can pose additional difficulties. ${ }^{2}$

Poor adherence has been linked to successive hospitalisation, increased need for medical interventions, morbidity and mortality. Furthermore, non-adherence results in increased healthcare costs, with North America having estimates of approximately $\$ 100$ billion being spent annually and $\$ 2000$ spent per patient per year for additional visits to the physician. ${ }^{19}$ It is necessary to verify if the use of mobile applications can help the patients to overcome these difficulties and improve treatment adherence. Despite the increased use of oral chemotherapy, the number of studies addressing the issue of adherence remains surprisingly low. ${ }^{20}$

\section{Objectives}

The aim of this systematic review and meta-analysis is to evaluate the effectiveness of mobile applications in improving adherence to oral chemotherapy and adjuvant hormonal therapy in cancer survivors.

\section{METHODS AND ANALYSIS}

This protocol is registered with the International Prospective Register of Systematic Reviews (PROSPERO). The Preferred Reporting Items for Systematic Reviews and Meta-Analyses (PRISMA) ${ }^{21}$ guidelines were used to design this systematic review protocol.

\section{Inclusion criteria}

This systematic review will include the following studies: those with randomised or quasi-experimental designs; those that include patients aged $>18$ years and those that evaluate the use of mobile applications by cancer survivors for adherence to oral chemotherapy and adjuvant hormonal therapy. There will be no language restrictions while selecting the studies.

\section{Patient, intervention, comparison and outcome strategy \\ - Patient: those undergoing oral chemotherapy or adju- vant hormonal therapy. \\ - Intervention: use of mobile applications. \\ - Comparator/control: no use of mobile applications. \\ - Outcome: improvement adherence to anticancer treatment.}

\section{Types of patients}

Studies where the patients are aged $>18$ years, diagnosed with cancer, undergoing oral chemotherapy or adjuvant hormonal therapy, and using mobile applications to improve treatment adherence will be included in this systemic review.

\section{Type of interventions}

Studies that compare the use of mobile applications with a concurrent control group to evaluate treatment adherence will be included in this systemic review.

\section{Type of outcome measures}

Non-adherence may lead to additional treatment costs due to the increased frequency of hospitalisation and medical appointments, recurrence of symptoms and consequent increase in drug toxicity caused by an overdose (to make up for the missed dose). ${ }^{422-25}$

The primary outcome will be to assess the improvement in treatment adherence. ${ }^{17}$ The secondary outcomes will be to assess the improvement in overall survival and life expectancy, improved quality of life and control of cancer-related symptoms. ${ }^{9-11}$

\section{Patient and public involvement}

This is a protocol for a systematic review and metaanalysis; the research will be conducted based on a wide and comprehensive literature search from relevant databases; the individual patient data will not be included. Thus, patients will not be involved while setting the search terms, in determining outcome measures, implementing study design and analysing the results.

\section{Search strategy}

The Cochrane Central Register of Controlled Trials, clinicaltrials.gov, Medline, Literatura latino-americana e do Caribe em Ciencias da Saúde (LILACS), Scopus and Embase will be used to search for articles published between January 2009 and July 2019. We selected the publications starting from January 2009 because the first drug reminder application was developed in 2009. ${ }^{56}$

The medical subject headings (MESH) terms will be: (antineoplastic agents OR oral anticancer agents OR drug therapy) AND (mobile application OR mobile apps OR app OR smartphone OR health informatics OR mobile health) AND (medication adherence OR patient empowerment OR treatment adherence and compliance) (table 1).

Eligible studies will also be selected from the reference lists of the retrieved articles.

\section{Data collection and analysis}

Selection of studies

Three authors, KSM, WAC and JFQ, will independently screen the search results using the titles and abstracts. Duplicate studies and reviews will be excluded. Two reviewers, KSM and MNM, will then go through the full text to determine whether the studies meet the inclusion criteria. Discrepancies will be resolved by a third reviewer, AKG. The selection of the studies is summarised in a PRISMA flow diagram (figure 1). 


\begin{tabular}{|ll}
\hline Table 1 & Medline search strategy \\
\hline Search items & \\
\hline 1 & Antineoplastic agents \\
\hline 3 & Oral anticancer agents \\
4 & Drug therapy \\
5 & OR/1-3 \\
6 & Mobile application \\
7 & Mobile apps \\
8 & Smartphone \\
9 & Health informatics \\
10 & Mobile health \\
11 & OR/5-9 \\
12 & Medication adherence \\
13 & Patient participation \\
14 & Patient compliance \\
15 & Treatment adherence and compliance \\
16 & Medication therapy management \\
17 & OR/11-15 \\
\hline
\end{tabular}

\section{Data extraction and management}

Various characteristics of the eligible studies will be extracted, including the first authors' last names, year of publication, location of the study (country), study design, primary objective, population, sample size, follow-up period, inclusion/exclusion criteria, type of mobile application used, type of control and primary results. Standardised data extraction forms will specifically be

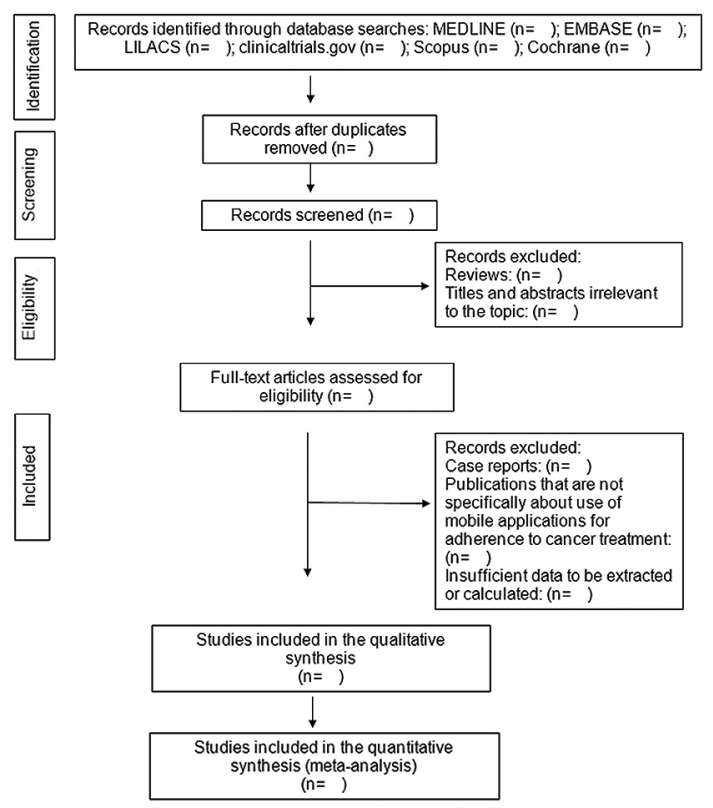

Figure 1 Preferred Reporting Items for Systematic Reviews and Meta-Analyses flow diagram. Flow diagram of the search for eligible studies in the use of mobile applications for adherence tocancer treatment: CENTRAL, Cochrane Central Register of Controlled Trials. created for this review and the results will be subsequently entered into a database. All data entries will be double-checked.

\section{Addressing missing data}

We will attempt to obtain any missing data by contacting the first or corresponding authors or coauthors of an article via phone, email or post. If we fail to receive any necessary information, the data will be excluded from our analysis and will be addressed in the discussion section.

\section{Risk-of-bias assessment}

Three authors, KSM, JFQ and BS, will independently assess the risk-of-bias in the eligible studies using the Cochrane risk-of-bias tool. ${ }^{25}$ The modified Cochrane Collaboration tool will be used to assess the risk-of-bias. Bias is assessed as a judgement (high, low or unclear) for individual elements from five domains (selection, performance, attrition, reporting and other).

\section{Assessment of heterogeneity}

The heterogeneity between study results will be evaluated using a standard $\chi^{2}$ test with a significance level of $\mathrm{p}<0.1$. To assess heterogeneity, we plan to compute the $\mathrm{I}^{2}$ statistic, which is a quantitative measurement of inconsistency across studies. A value of $0 \%$ indicates no heterogeneity, whereas $\mathrm{I}^{2}$ values $\geq 50 \%$ indicate a substantial level of heterogeneity; however, heterogeneity will be assessed only if it is appropriate to conduct a meta-analysis.

\section{Analysis}

Data will be entered into the Review Manager software (RevMan5.2.3). This software allows the user to enter protocols; complete reviews; include text, characteristics of the studies, comparison tables and study data; and perform meta-analyses. For dichotomous outcomes, we will extract or calculate the OR and 95\% CI for each study. In case of heterogeneity $\left(\mathrm{I}^{2} \geq 50 \%\right)$, the random-effects model will be used to combine the studies to calculate the OR and 95\% CI, using the DerSimonian-Laird algorithm in the meta for package, which provides functions for conducting meta-analyses in R.

Other study characteristics and results will be summarised narratively if the meta-analysis cannot be performed for all or some of the included studies. Sensitivity analyses will be used to explore the robustness of the findings regarding the study quality and sample size. This is only possible if we can conduct a meta-analysis. Sensitivity analyses will be shown in a summary table.

\section{Grading quality of evidence}

For grading the strength of evidence from the included data, we will use the Grading of Recommendation Assessment, Development, and Evaluation approach. The summary of the assessment will be incorporated into broader measurements to ensure the judgement on the risk-of-bias, consistency, directness and precision. ${ }^{26}$ 


\section{DISCUSSION}

Non-adherence to cancer treatment is a very common and relevant clinical problem, with a significant adverse impact on the healthcare system. In this systematic review and meta-analysis, we aim to determine the effect of mobile applications on the improvement of treatment adherence in cancer survivors. In theory, mobile applications can improve adherence to anticancer treatment, because they can remind the patient to take the medicine on time and assist in care management. ${ }^{27} 28$ We expect that our study will provide accurate data to develop effective strategies for adherence to anticancer treatment and help to improve our understanding of the role of mobile applications in this context.

\section{Ethics and dissemination}

Ethical approval is not required because this systematic review will use the published data. Findings of this systematic review will be published in a peer-reviewed journal and will be updated if there is enough new evidence to change the conclusions of the systematic review.

Acknowledgements The authors acknowledge the assistance provided by the Graduate Program in Health Sciences of the Federal University of Rio Grande do Norte (UFRN) in conducting the literature search.

Contributors KSM, BS and AKG designed this systematic review and metaanalysis. KSM drafted the manuscript, and AKG revised it. KSM, RNC and AKG developed the search strategies and KSM, JFQ and MNM will implement it. KSM, MNM, JFQ and WAC will track potential studies, extract data and assess the quality; in case of disagreement between the authors, AKG will advise on the methodology and will be the referee. RNC will complete the data synthesis. All authors have approved the final version of this manuscript.

Funding The authors have not declared a specific grant for this research from any funding agency in the public, commercial or not-for-profit sectors.

Competing interests None declared.

Patient consent for publication Not required.

Provenance and peer review Not commissioned; externally peer reviewed.

Open access This is an open access article distributed in accordance with the Creative Commons Attribution Non Commercial (CC BY-NC 4.0) license, which permits others to distribute, remix, adapt, build upon this work non-commercially, and license their derivative works on different terms, provided the original work is properly cited, appropriate credit is given, any changes made indicated, and the use is non-commercial. See: http://creativecommons.org/licenses/by-nc/4.0/.

\section{REFERENCES}

1 Weingart SN, Flug J, Brouillard D, et al. Oral chemotherapy safety practices at US cancer centres: questionnaire survey. BMJ 2007;334.

2 Wood L. A review on adherence management in patients on oral cancer therapies. Eur J Oncol Nurs 2012;16:432-8.

3 Moody M, Jackowski J. Are patients on oral chemotherapy in your practice setting safe? Clin J Oncol Nurs 2010;14:339-46.

4 Chabrier M, Bezy O, Mouret M-A, et al. Impact of depressive disorders on adherence to oral anti-cancer treatment. Bull Cancer 2013;100:1017-22.

5 Burhenn P, Smudde J. Using tools and technology to promote education and adherence to oral agents for cancer. Clin $\mathrm{J}$ Oncol Nurs 2015;19:53-9.
6 Greer JA, Amoyal N, Nisotel L, et al. A systematic review of adherence to oral antineoplastic therapies. Oncologist 2016;21:354-76.

7 Hershman DL, Kushi LH, Hillyer GC, et al. Psychosocial factors related to non-persistence with adjuvant endocrine therapy among women with breast cancer: the breast cancer quality of care study (BQUAL). Breast Cancer Res Treat 2016;157:133-43.

8 Mathes T, Antoine S-L, Pieper D, et al. Adherence enhancing interventions for oral anticancer agents: a systematic review. Cancer Treat Rev 2014;40:102-8.

9 Ali EE, Chan SSL, Leow JL, et al. User acceptance of an app-based adherence intervention: perspectives from patients taking oral anticancer medications. J Oncol Pharm Pract 2019;25:390-7.

10 Ali EE, Chan SSL, Poh HY, et al. Design Considerations in the Development of App-Based Oral Anticancer Medication Management Systems: a Qualitative Evaluation of Pharmacists' and Patients' Perspectives. J Med Syst 2019;43:63.

11 Fishbein JN, Nisotel LE, MacDonald JJ, et al. Mobile application to promote adherence to oral chemotherapy and symptom management: a protocol for design and development. JMIR Res Protoc 2017;6:e62

12 Neuss MN, Polovich M, McNiff K, et al. 2013 updated American Society of clinical Oncology/Oncology nursing Society chemotherapy administration safety standards including standards for the safe administration and management of oral chemotherapy. J Oncol Pract 2013;9:5s-13.

13 Darlow S, Wen K. Development testing of mobile health interventions for cancer patient self-management: a review. Health Informatics $J$ 2015;27:1-18.

14 Jacobs JM, Pensak NA, Sporn NJ, et al. Treatment satisfaction and adherence to oral chemotherapy in patients with cancer. JOP 2017;13:e474-85.

15 Medicine, U.S. National library of medicine. Medical subject Headings: mobile applications, 2018. Available: <https://www.ncbi. nlm.nih.gov/mesh/68063731> [Accessed 16 Mar 2019].

16 Elliott M, Liu Y. The nine rights of medication administration: an overview. Br J Nurs 2010;19:300-5.

17 Zerillo JA, Goldenberg BA, Kotecha RR, et al. Krzyzanowska MK interventions to improve oral chemotherapy safety and quality: a systematic review. JAMA Oncol 2018;4:105-17.

18 Makubi A, Sasi P, Ngaeje M, et al. Rationale and design of mDOTHuA study: a randomized trial to assess the effect of mobiledirectly observed therapy on adherence to hydroxyurea in adults with sickle cell anemia in Tanzania. BMC Med Res Methodol 2016;16:140.

19 Thakkar J, Kurup R, Laba T-L, et al. Mobile telephone text messaging for medication adherence in chronic disease. JAMA Intern Med 2016;176:340-9.

20 Barillet M, Prevost V, Joly F, et al. Oral antineoplastic agents: how do we care about adherence? Br J Clin Pharmacol 2015;80:1289-302.

21 Moher D, Liberati A, Tetzlaff J, et al. Preferred reporting items for systematic reviews and meta-analyses: the PRISMA statement. PLoS Med 2009;6:e1000097.

22 Shimada CS. Adherence to treatment with oral medications. Rio de Janeiro: Elsevier, 2016.

23 Ruddy K, Mayer E, Partridge A. Patient adherence and persistence with oral anticancer treatment. CA Cancer J Clin 2009;59:56-66.

24 Jin J, Sklar GE, Min Sen Oh V, et al. Factors affecting therapeutic compliance: a review from the patient's perspective. Ther Clin Risk Manag 2008;4:269-86.

25 Higgins JP, Altman DG, Gøtzsche PC, et al. The Cochrane collaboration's tool for assessing risk of bias in randomised trials. Cochrane bias methods group; Cochrane statistical methods group. BMJ 2011;343.

26 Balshem H, Helfand M, Schünemann HJ, et al. Grade guidelines: 3. rating the quality of evidence. J Clin Epidemiol 2011;64:401-6.

27 Jupp JCY, Sultani H, Cooper CA, et al. Evaluation of mobile phone applications to support medication adherence and symptom management in oncology patients. Pediatr Blood Cancer 2018;65:e27278.

28 Carmody JK, Denson LA, Hommel KA. Content and usability evaluation of medication adherence mobile applications for use in pediatrics. J Pediatr Psychol 2019;44:333-42. 